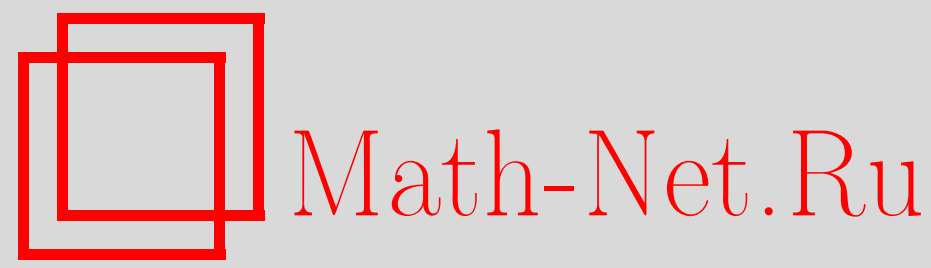

Д. А. Камаев, Семейства устойчивых многообразий одномерных параболических уравнений, $M a$ тем. заметки, 1996, том 60, выпуск 1, 11-23

DOI: https://doi.org/10.4213/mzm1799

Использование Общероссийского математического портала MathNet.Ru подразумевает, что вы прочитали и согласны с пользовательским соглашением

http://www . mathnet.ru/rus/agreement

Параметры загрузки:

IP : 35.173 .219 .12

26 апреля 2023 г., 12:49:43

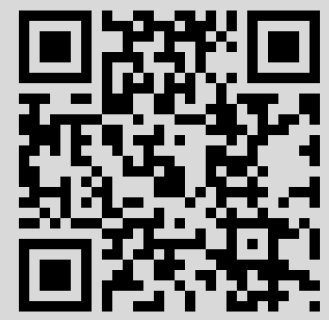


том 60 выпуск 1 июль 1996

\title{
СЕМЕЙСТВА УСТОЙЧИВЫХ МНОГООБРАЗИЙ ОДНОМЕРНЫХ ПАРАБОЛИЧЕСКИХ УРАВНЕНИЙ
}

\author{
Д. А. Камаев
}

1. Введение. Одной из основных проблем теории эволюционных дифференциальных уравнений является исследование асимптотического поведения траектории последних. Эта проблема решается, в частности, при помощи построения инвариантных семейств многообразий. В настоящей заметке доказывается существование семейства устойчивых многообразий у инвариантного множества динамической системы, порождаемой одномерным параболическим уравнением

$$
u_{t}=-L u+B(u, t), \quad u\left(t_{0}, x\right)=\varphi(x), \quad x \in(0,1),
$$

с периодическими или однородными краевыми условиями I, II, III типа. Оператор $L$ с гладкими коэффициентами удовлетворяет условию эллиптичности [1] и определяется соотношением $L u=-\left(d(x) u_{x}\right)_{x}-a(x) u$, а оператор $B(u, t)$ задается выражением $B(u, t)=f\left(u, u_{x}, x, t\right)$. Функция $f(\xi, \zeta, x, t)$ предполагается гладкой, причем для любых неотрищательных целых чисел $k, l, m, n$ существует непрерьвная неубьвающая функция $\alpha_{k, l, m, n}(s) \geqslant 0$ такая, что

$$
\left|\frac{\partial^{k+l+m+n} f(\xi, \zeta, x, t)}{\partial \xi^{k} \partial \zeta^{l} \partial x^{m} \partial t^{n}}\right| \leqslant \alpha_{k, l, m, n}(|\xi|)(1+|\zeta|) .
$$

Вопрос о существовании инвариантных многообразий бесконечномерных динамических систем, порождаемых уравнениями вида (1), рассматривался в ряде работ [2]-[4] в предположении, что $f$ от $u_{x}$ не зависит. В настоящей заметке это ограничение снято. Для сокращения изложения рассматриваются автономные уравнения. Это упрощение не является принципиальным, все результаты без труда обобщаются на неавтономный случай. Фиксируем для определенности однородные краевые условия I рода.

2. Динамическая система. Рассмотрим гильбертово пространство $W$ квадратично интегрируемых функций, определенных на отрезке $[0,1]$. Пространство $W$ снабжено стандартным скалярньм произведением $(\cdot, \cdot)$, 
порождающим норму $|\cdot|$ (см. [1]). Оператор $L$ имеет в $W$ всюду плотную область определения и является самосопряженным. Существует ортонормированньй базис $\left\{v_{k}(x)\right\}, k \in \mathbb{N}$, в $W$ такой, что $L v_{k}=\lambda_{k}^{2} v_{k}, \lambda_{k}^{2}<\lambda_{k+1}^{2}$, $\lambda_{k}^{2} \rightarrow \infty$.

Для каждого натурального $k$ определим гильбертово пространство $W^{k}$ как пополнение области определения оператора $L^{k / 2}$ относительно нормы $|u|_{k}=\left|L^{k / 2} u\right|$, порождаемой скалярным произведением $(u, v)_{k}=$ $\left(L^{k / 2} u, L^{k / 2} v\right)$. По определению $W^{0}=W, W^{\infty}=\bigcap_{k \geqslant 1} W^{k}$. В силу теоремы Реллиха естественное вложение $W^{k} \rightarrow W^{k-1}$ компактно.

С задачей (1) связаны еще три пространства: $L_{2}(t, s ; W), W_{t, s}$ и $H_{t, s}$. Элементами первого являются измеримые по $t$ функции $u(t)$ со значениями в $W$, имеющие конечную норму $\|u\|_{t, s}$, порожденную скалярным произведением $(u, v)_{t, s}=\int_{t}^{s}(u(\tau), v(\tau)) d \tau$. Банахово пространство $W_{t, s}$ состоит из элементов $u(t) \in L_{2}(t, s ; W)$, для которых $d u / d t, L u$ суть элементы $L_{2}(t, s ; W)$, а $L^{1 / 2} u(\tau)$ при $\tau \in[t, s]$ есть элемент $W^{1}$, непрерывно зависящий от $\tau$ в норме пространства $W^{1}$, и выполняется соотношение

$$
\left(\frac{d u}{d t}(\tau), L u(\tau)\right)=\frac{1}{2} \frac{d}{d t}|u(\tau)|_{1}^{2} \in L_{2}(t, s) .
$$

Норма в $W_{t, s}$ определяется формулой

$$
\|u\|_{W_{t, s}}=\left[\|L u\|_{t, s}^{2}+\left\|u_{t}\right\|_{t, s}^{2}+\sup _{\tau \in[t, s]}|u(\tau)|_{1}^{2}\right]^{1 / 2} .
$$

Для элементов пространства $W_{t, s}$ рассмотрим более слабую норму

$$
\|u\|_{H_{t, s}}=\left[\int_{t}^{s}|u(\tau)|_{1}^{2} d \tau+\sup _{\tau \in[t, s]}|u(\tau)|^{2}\right]^{1 / 2} .
$$

Пополнение пространства $W_{t, s}$ относительно этой нормы есть пространство $H_{t, s}$.

Как известно, для решений линейной задачи Коши

$$
u_{t}=-L u+f(t), \quad u\left(t_{0}\right)=\varphi,
$$

где $f(t) \in L_{2}\left(t_{0}, s ; W\right), \varphi \in W^{1}$, имеет место априорная оценка

$$
\|u\|_{W_{\tau, s}}^{2} \leqslant 2\left(|u(\tau)|_{1}^{2}+\|f(t)\|_{\tau, s}^{2}\right), \quad \tau \in\left[t_{0}, s\right] .
$$

Если в задаче (1) $\varphi \in W^{1}$, то при помощи априорной оценки (2) и метода последовательных приближений можно доказать [5] существование величины $t\left(\left|\varphi_{1}\right|\right)>0$, зависящей лишь от $|\varphi|_{1}$, и единственного элемента $u(t ; \varphi) \in W_{0, t\left(|\varphi|_{1}\right)}, u(0 ; \varphi)=\varphi$, которьй является обобшенным решением задачи (1). Обозначим через $T(\varphi)$ длину максимального интервала, на котором существует обобщенное решение. В качестве фазового пространства для задачи (1) выберем $W^{1}$. 
ОПредЕЛЕниЕ. Ограниченное замкнутое подмножество $\Lambda \subset W^{1}$ называется инвариантным для задачи Коши (1), если для любого $\varphi \in \Lambda$ $u(t ; \varphi) \in \Lambda$ при всех $t \in[0, T(\varphi)]$.

Если $\Lambda$ - инвариантное множество, то $T(\varphi)=\infty$ для любого $\varphi \in \Lambda$. Следовательно, уравнение (1) порождает на $\Lambda$ динамическую систему с непрерьвньп временем: $F_{t}(\varphi)=u(t, \varphi), t \geqslant 0$.

Рассмотрим бесконечно гладкую функцию $0 \leqslant h_{\rho}(s) \leqslant 1$, причем $h_{\rho}(s)=1$, если $s \leqslant \rho$, и $h_{\rho}(s)=0$, если $s>2 \rho$. При изучении поведения траекторий задачи (1) в окрестности $\mathscr{U}$ инвариантного множества $\Lambda$, $\mathscr{U} \subset \mathscr{B}_{\rho}=\left\{u \in W^{1}:|u|_{1}<\rho\right\}$, без ограничения обшности можно заменить в уравнении (1) оператор $B(u)$ оператором $B_{1}(u)=h_{\rho}\left(|u|_{1}\right) B(u)$. Такая замена не изменит поведения траекторий внутри $\mathscr{B} \rho$. Однако, измененное уравнение порождает во всем $W^{1}$ динамическую систему с непрерьвньм временем. Далее всюду предполагается, что указанная замена проделана для фиксированного инвариантного множества $\Lambda$, его окрестности $\mathscr{U} \subset \mathscr{B} \rho$ и величины $\rho$.

Пусть $\operatorname{End}\left(W^{1}\right)$ - банахово пространство непрерьвных линейных отображений $W^{1}$ в себя. Дифференциальные свойства динамической системы, порождаемой уравнением (1), описываются теоремой 1. Ее доказательство стандартно (см. [5]) и поэтому опущено.

Теорема 1. Если $t \geqslant 0$, то отображсение $F_{t}$ имеет непрерывную производную Фреше $\mathscr{D}_{\varphi} F_{t}, \varphi \in W^{1}$, причем $\mathscr{D}_{\varphi} F_{t}(\theta)=v(t ; \theta)$, где $v(t ; \theta)$ - решение линеаризованного вдоль траектории $u(t ; \varphi)$ уравнения (1) с однородными краевыми условиями I рода. Существует непрерывная неубывающая по $t$ функция $l(\rho, t)>0$ такая, что $|v(t ; \theta)|_{1} \leqslant l(\rho, t)|\theta|_{1}$. Отображсение $\mathscr{D}_{\varphi} F_{t}: \varphi \mapsto \mathscr{D}_{\varphi} F_{t}$, переводящее $W^{1}$ в $\operatorname{End}\left(W^{1}\right)$, удовлетворяет условию Липшица с константой $L(\rho, t)$, непрерывно зависящей от $(\rho, t)$ и неубывающей по $t$. Для любого ограниченного подмножества $M \subset W^{1}$ множество $F_{t}(M)$ ограничено в $W^{k}, t>0, k \in \mathbb{N}$. Если $t>0$, то при $s \rightarrow t$ величины $\left|F_{t}(\varphi)-F_{s}(\varphi)\right|_{1}$ равномерно по $\varphi \in \Lambda$ стремятся $к$ нулю.

3. Оценки решений задачи Коши. В дальнейших рассуждениях нам понадобится оценка величины $w(t ; \varphi)=u(t ; \varphi)-\varphi$ в норме пространства $W_{0, t}$.

ЛЕмма 1. Существуют константы $a_{1}\left(|\varphi|_{2}\right), a_{2}\left(|\varphi|_{3}\right)$, определяемые функцией $f$, величиной $\rho$ и непрерывно зависящие от $|\varphi|_{2},|\varphi|_{3}$, такие, что для $0 \leqslant t \leqslant 1, \varphi \in \Lambda$, имеют место неравенства

$$
\begin{aligned}
\|w(t ; \varphi)\|_{W_{0, t}} & \leqslant a_{1}\left(|\varphi|_{2}\right) \sqrt{t}, \\
\left\|L^{1 / 2} w(t ; \varphi)\right\|_{W_{0, t}} & \leqslant a_{2}\left(|\varphi|_{3}\right) \sqrt{t} .
\end{aligned}
$$


ДоКАЗАТЕЛЬСТво. Подставим в (1) $w=u-\varphi$ и применим к полученному уравнению относительно $w$ априорную оценку (2). В результате получим неравенство

$$
\|w(t ; \varphi)\|_{W_{0, t}}^{2} \leqslant 4\left(\left\|B_{1}(u)\right\|_{0, t}^{2}+|\varphi|_{2}^{2} t\right) .
$$

Величину $\left\|B_{1}(u)\right\|_{0, t}^{2}$ оценим при помоши неравенства $\sup _{x}|u(x)| \leqslant c|u|_{1}$, $u \in W^{1}, c$ - константа [1], воспользовавшись ограниченностью инвариантного множества $\Lambda$ в $W^{2}$ и гладкостью функции $f$. В итоге получим первое неравенство леммы 1. Аналогично доказывается второе неравенство.

Рассмотрим семейство спектральных задач с однородньми краевыми условиями I рода:

$$
\begin{gathered}
L_{\varphi} v \equiv d(x) v_{x x}+\left(d^{\prime}(x)+f_{u_{x}}(\varphi, x)\right) v_{x} \\
+\left(a(x)+f_{u}(\varphi, x)\right) v=\lambda v,
\end{gathered}
$$

где $\varphi \in \Lambda, f_{u}(\varphi, x)=f_{u}\left(\varphi(x), \varphi_{x}(x), x\right), f_{u_{x}}(\varphi, x)=f_{u_{x}}\left(\varphi(x), \varphi_{x}(x), x\right)$. Очевидно, $L_{\varphi}$ есть линеаризация оператора правой части уравнения (1) в точке $\varphi \in \Lambda$. Обозначим собственные числа и собственные функции задачи (3) через $\lambda_{n}(\varphi), v_{n}(\varphi, x), \lambda_{n+1}(\varphi)<\lambda_{n}(\varphi), n \in \mathbb{N}$.

Если $v$ - решение задачи (3), то функция $\eta(z)$, связанная с $v$ преобразованием Лиувилля $v=E_{\varphi}(\eta)$ (см. [6]), удовлетворяет следующей спектральной задаче с нулевьми краевыми условиями I рода:

$$
\eta_{z z}+q(\varphi, z) \eta=\mu \eta, \quad z \in(0,1) .
$$

В данном случае преобразование $E_{\varphi}$ задается формулами

$$
\begin{gathered}
z=\lambda_{0}^{-1} \int_{0}^{x}(d(s))^{-1 / 2} d s, \quad \eta=(r(x) p(x))^{1 / 4} v \\
\mu=\lambda_{0} \lambda, \quad \lambda_{0}=\int_{0}^{1}(d(s))^{-1 / 2} d s
\end{gathered}
$$

где

$$
p(x)=\exp \left(\int_{0}^{x}\left[d^{\prime}(s)+f_{u_{x}}(\varphi, s)\right](d(s))^{-1} d s\right), \quad r(x)=p(x)(d(x))^{-1} .
$$

При этом

$q(\varphi, z)=-\theta^{\prime \prime}(z)(\theta(z))^{-1}-\lambda_{0}^{2}\left(a(x)+f_{u}(\varphi, x)\right), \quad \theta(z)=(r(x) p(x))^{1 / 4}$. 
Учитьвая асимптотику собственных функций и собственных значений спектральной задачи (4) [6], получаем равномерные по $\varphi \in \Lambda(\Lambda-$ ограничено в $W^{3}$ ) асимптотические формулы

$$
\begin{aligned}
\lambda_{n}(\varphi) & =-\frac{1}{\pi^{2} \lambda_{0}}\left(n+\frac{\alpha_{1}}{n}+O\left(n^{-2}\right)\right)^{2}, \quad \alpha_{1}=\frac{\pi^{2}}{2} \int_{0}^{1} q(\varphi, z) d z, \\
v_{n}(\varphi, x) & =E_{\varphi}\left(\sqrt{\frac{2}{\pi}} \sin (\pi n z)+O\left(n^{-1}\right)\right) .
\end{aligned}
$$

Соотношения (5) позволяют утверждать, что $E_{\varphi} \in \operatorname{End}\left(W^{1}\right)$. Кроме того, сушествуют положительные не зависяшие от $\varphi \in \Lambda$ константы $\delta_{1}, \delta_{2}$, для которых $\delta_{1}|v|_{1} \leqslant\left|E_{\varphi} v\right|_{1} \leqslant \delta_{2}|v|_{1}, v \in W^{1}$.

Введем семейство билинейных форм на $W^{1}$ :

$$
[u, v]_{(\varphi)}=\int_{0}^{1}\left\{\left(E_{\varphi}^{-1} v\right)_{z}\left(E_{\varphi}^{-1} u\right)_{z}-(q(\varphi, z)-\gamma)\left(E_{\varphi}^{-1} u\right)\left(E_{\varphi}^{-1} v\right)\right\} d z
$$

параметр $\gamma>0, \varphi \in \Lambda$.

ЛЕмМА 2. При достаточно большом $\gamma$, не зависящем от $\varphi \in \Lambda$, билинейные формы (8) яв.яются скалярными произведениями, относительно которых ортогональны собственные функиии задачи (3). Кроме того, существуют положительные не зависящие от $\varphi \in \Lambda$ константы $\beta_{1}, \beta_{2}$ такие, что для любого $v \in W^{1} \beta_{1}|v|_{1} \leqslant|v|_{(\varphi)} \leqslant \beta_{2}|v|_{1}$, əде $|v|_{(\varphi)}=\sqrt{[v, v]_{(\varphi)}}$.

ДокАЗАТЕЛЬСтво. Утверждения леммы вытекают из ограниченности $\Lambda$ в $W^{3}$, определения преобразования $E_{\varphi}$, формул (6), свойств спектральной задачи (4) [6] и неравенства $\sup _{x}|u(x)| \leqslant c|u|_{1}, u \in W^{1}, c-$ константа [1].

В дальнейшем $\gamma$ считается фиксированным и удовлетворяющим условию леммы 2.

Обозначим через $H_{n}(\varphi)$ собственное подпространство, натянутое на функции $v_{1}(\varphi, x), \ldots, v_{n}(\varphi, x)$. Соответствуюшее ему дополнительное подпространство $H_{n}^{\prime}(\varphi)$ натянуто на функции $v_{k}(\varphi, x), k>n$. Пусть для $\varphi \in \Lambda \pi_{n}(\varphi): W \rightarrow H_{n}(\varphi), \pi_{n}^{\prime}(\varphi): W \rightarrow H_{n}^{\prime}(\varphi)$ есть проекторы, задаваемые прямой суммой $H_{n}(\varphi) \oplus H_{n}^{\prime}(\varphi)$, которая ортогональная относительно скалярного произведения (8).

Лемма 3. Существуют не зависящие от $\varphi \in \Lambda$ константы $c_{i} \geqslant 0$, $i=1,2,3,4$, такие, что при $\varphi \in \Lambda, t \in[0,1]$ справедливы неравенства

$$
\begin{array}{lll}
\left|\pi_{n}(\varphi) \mathscr{D}_{\varphi} F_{t}(\theta)\right|_{(\varphi)} \geqslant\left(e^{\left(\lambda_{n}(\varphi)-\gamma \lambda_{0}^{-1}\right) t}-t c_{1}\right)|\theta|_{(\varphi)}, & & \theta \in H_{n}(\varphi) ; \\
\left|\pi_{n}^{\prime}(\varphi) \mathscr{D}_{\varphi} F_{t}(\theta)\right|_{(\varphi)} \leqslant\left(e^{\left(\lambda_{n+1}(\varphi)-\gamma \lambda_{0}^{-1} t\right)}+t c_{2}\right)|\theta|_{(\varphi)}, & & \theta \in H_{n}^{\prime}(\varphi) ; \\
\left|\pi_{n}^{\prime}(\varphi) \mathscr{D}_{\varphi} F_{t}(\theta)\right|_{(\varphi)} \leqslant c_{3} t|\theta|_{(\varphi)}, & \theta \in H_{n}(\varphi) ; \\
\left|\pi_{n}(\varphi) \mathscr{D}_{\varphi} F_{t}(\theta)\right|_{(\varphi)} \leqslant c_{4} t|\theta|_{(\varphi)}, & \theta \in H_{n}^{\prime}(\varphi) .
\end{array}
$$


ДокАЗАТЕЛЬСтво. Все четыре неравенства доказьваются аналогично. Докажем, к примеру, первое. Для этого представим линеаризованную задачу (1) в интегральной форме:

$$
v(t)=\exp (t S(\varphi)) \theta+\int_{0}^{t} \exp ((t-s) S(\varphi))\left[J(s) v(s)+\gamma \lambda_{0}^{-1} v(s)\right] d s,
$$

где оператор $S(\varphi)$ определяется выражением

$$
\begin{gathered}
S(\varphi) v=L_{\varphi} v-\gamma \lambda_{0}^{-1} v \\
J(t) v=\left(f_{u}\left(u, u_{x}, x\right)-f_{u}(\varphi, x)\right) v+\left(f_{u_{x}}\left(u, u_{x}, x\right)-f_{u_{x}}(\varphi, x)\right) v_{x}, \\
u=F_{t}(\varphi), \quad v=\mathscr{D}_{\varphi} F_{t}(\theta) .
\end{gathered}
$$

Применим к соотношению (9) оператор $\pi_{n}(\varphi)$, а затем оценим снизу правую часть, воспользовавшись теоремой 1 и леммой 2 . В результате получим

$$
\begin{aligned}
& \left|\pi_{n}(\varphi) v(t)\right|_{(\varphi)} \geqslant \exp \left(\lambda_{n}(\varphi) t-\gamma \lambda_{0}^{-1}(t)\right)|\theta|_{(\varphi)} \\
& -\beta_{2}\left|\int_{0}^{t} \exp ((t-s) S(\varphi)) J(s) v(s) d s\right|_{1}-\gamma \lambda_{0}^{-1} \beta_{2} \beta_{1}^{-1} l(\rho, 1) t|\theta|_{(\varphi)} .
\end{aligned}
$$

Для решения $w$ задачи Коши

$$
w_{t}=S(\varphi) w+g(t), \quad w(0)=0, \quad g(t) \in L_{2}(0,1 ; W),
$$

справедлива априорная оценка

$$
\|w(t)\|_{W_{0, t}}^{2} \leqslant c_{0}\|g(t)\|_{0, t}^{2}, \quad t \in[0,1]
$$

которая получается из (11) при помощи априорной оценки (2) с учетом ограниченности $\Lambda$ в $W^{2}$. Стандартные рассуждения [1], [5] позволяют утверждать, что константа $c_{0}>0$ не зависит от $\varphi$, а определяется функциями $f_{u}, f_{u_{x}}$, величиной $\gamma \lambda_{0}^{-1}$ и множеством $\Lambda$.

Вернемся к неравенству (10). Полагая в неравенстве (12) $g=J(t) v$, получаем оценку первого вычитаемого в правой части (10):

$$
\left|\int_{0}^{t} \exp ((t-s) S(\varphi)) J(s) v(s) d s\right|_{1}^{2} \leqslant c_{0}\|J(s) v(s)\|_{0, t}^{2} .
$$

Воспользовавшись формулой конечных приращений и ограниченностью $\Lambda$ в $W^{2}$, можно доказать существование не зависящей от $\varphi$ константы $c_{0,1}>0$, которая определяется производными функции $f$ и множеством $\Lambda$, такой, что

$$
\|J(s) v(s)\|_{0, t}^{2} \leqslant c_{0,1}^{2}\left\{\left\|w_{x} v\right\|_{0, t}^{2}+\left\|v_{x} w\right\|_{0, t}^{2}+\|v w\|_{0, t}^{2}+\left\|v_{x} w_{x}\right\|_{0, t}^{2}\right\} .
$$


В этом неравенстве $w(t)=F_{t}(\varphi)-\varphi$. Теперь нам необходимо оценить слагаемые в правой части (14). Все они оцениваются одинаково. Оценим, к примеру, последнее. В силу теоремы вложения [1], теоремы 1 и леммы 1 получаем

$$
\left\|v_{x} w_{x}\right\|_{0, t}^{2} \leqslant c_{0,2}^{2} \int_{0}^{t}\left\|L^{1 / 2} w\right\|_{W_{0, s}}^{2}\left|v_{x}(s)\right|^{2} d s \leqslant c_{0,3}^{2} t^{2}|\theta|_{1}^{2},
$$

где константы $c_{0,2}>0, c_{0,3}>0$ определяются функциями $d(x), a(x)$ и величинами $l(\rho, 1), \sup _{\varphi \in \Lambda} a_{2}\left(|\varphi|_{3}\right)$. Таким образом, для некоторой константы $c_{0}^{\prime}>0$ имеет место неравенство

$$
\left|\int_{0}^{t} \exp ((t-s) S(\varphi)) J(s) v(s) d s\right|_{1} \leqslant c_{0}^{\prime} t|\theta|_{1} .
$$

Из неравенств (10) и (15) получаем первое неравенство из формулировки леммы 3 .

4. Семейство устойчивых многообразий. Лемма 3 позволяет доказать сушествование у инвариантного множества $\Lambda$ инвариантного семейства экспоненциально сжимающихся под действием $F_{t} C^{1}$-подмногообразий (семейства устойчивых многообразий).

ТЕОрема 2. Пусть $\Lambda$-инвариантное множество динамической системы $F_{t}, t \geqslant 0$, порождаемой уравнением (1). Тогда существует $M \in \mathbb{N}$ и невозрастающая последовательность $\varepsilon_{n} \rightarrow 0, n \rightarrow \infty, n \in \mathbb{N}$, такие, что при $n \geqslant M$ имеют место следующие утверждения.

1. Существует $C^{1}$-непрерывное семейство $\left\{W_{\varepsilon_{n}}^{s}(\varphi), \varphi \in \Lambda\right\}$ $C^{1}$-подмногообразий, причем через каждую точку $\varphi \in \Lambda$ проходит ровно одно подмногообразие $W_{\varepsilon_{n}}^{s}(\varphi)$.

2. Отобрахсение $\pi_{n}^{\prime}(\varphi): W_{\varepsilon_{n}}^{s}(\varphi) \rightarrow H_{n}^{\prime}(\varphi)$ есть диффеоморфизм, причем $\mathscr{D}_{\varphi} \pi_{n}^{\prime}(\varphi)=\mathrm{id} u$

$$
\pi_{n}^{\prime}(\varphi)\left(W_{\varepsilon_{n}}^{s}(\varphi)\right)=\left\{\psi: \psi=\pi_{n}^{\prime}(\varphi)(\varphi+\theta),|\theta|_{(\varphi)}<\varepsilon_{n}\right\}, \quad \varphi \in \Lambda .
$$

3. Семейство $\left\{W_{\varepsilon_{n}}^{s}(\varphi), \varphi \in \Lambda\right\}$ инвариантно:

$$
F_{t}\left(W_{\varepsilon_{n}}^{s}(\varphi)\right) \subset W_{\varepsilon_{n}}^{s}\left(F_{t}(\varphi)\right), \quad t \geqslant 0, \quad \varphi \in \Lambda .
$$

4. Многообразия $W_{\varepsilon_{n}}^{s}(\varphi), \varphi \in \Lambda$, экспоненциально сжсимаются под действием $F_{t}$, т.е. существуют не зависящие от $\varphi$ константы $A>0, \alpha_{n}>0, \alpha_{n} \rightarrow \infty$, такие, что для любых $\varphi_{1}$, $\varphi_{2} \in W_{\varepsilon_{n}}^{s}(\varphi), t \geqslant 0$

$$
\left|F_{t}\left(\varphi_{1}\right)-F_{t}\left(\varphi_{2}\right)\right|_{1} \leqslant A e^{-\alpha_{n} t}\left|\varphi_{1}-\varphi_{2}\right|_{1} .
$$

5. $Е с л и \varphi_{1} \in W_{\varepsilon_{n}}^{s}(\varphi) \cap \Lambda, \operatorname{mo} \varphi_{1}=\varphi$. 
ДокАЗАТЕльство. По теореме 1 множество $\Lambda$ ограничено в $W^{k}, k \in \mathbb{N}$. Следовательно, для любого $k \Lambda$-компактноеподмножество $W^{k}$ и топологии, индуцированные на $\Lambda$ вложениями $\Lambda \rightarrow W^{k}, \Lambda \rightarrow W^{k+1}$, совпадают. Так как $\Lambda$ состоит из гладких функций, то ограничения $F_{t} \mid \Lambda, t \geqslant 0$, являются гомеоморфизмами и уравнение (1) порождает на $\Lambda$ непрерьвную динамическую систему $F_{t}, t \in \mathbb{R}($ см. $[7, \S 2])$.

Доказательство теоремы опирается на технику преобразования графиков (см. $[8, \S 5,4],[3])$.

Рассмотрим семейство разложений

$$
W^{1}=H_{n}(\varphi) \oplus H_{n}^{\prime}(\varphi), \quad \varphi \in \Lambda .
$$

Из свойства непрерывной зависимости спектральной задачи (4) от $q(\varphi, z)$ и определения преобразования $E_{\varphi}$ вытекает непрерьвность семейства (16) по $\varphi \in \Lambda$ [6]. Разложения (16) индуцируют разложение касательного расслоения $T_{\Lambda} W^{1}$ в сумму Уитни $T_{\Lambda} W^{1}=H_{n}(\Lambda) \oplus H_{n}^{\prime}(\Lambda)$.

Обозначим через $\Sigma_{c}(\Lambda)$ множество непрерывных сечений расслоения $T_{\Lambda} W^{1}$. Прямая сумма подпространств $\Sigma_{n}, \Sigma_{n}^{\prime}$, состоящих из непрерывных сечений вида $\pi_{n}(\varphi) \sigma(\varphi), \pi_{n}^{\prime}(\varphi) \sigma(\varphi), \varphi \in \Lambda, \sigma \in \Sigma_{c}(\Lambda)$, совпадает с $\Sigma_{c}(\Lambda)$. В приведенных обозначениях и всюду далее принято каноническое отождествление слоев $T_{\varphi} W^{1}$ расслоения $T_{\Lambda} W^{1}$ с пространством $W^{1}$, а слоев расслоений $H_{n}(\Lambda), H_{n}^{\prime}(\Lambda)$ с подпространствами пространства $W^{1}$.

Пусть

$$
\Sigma_{n}(\Lambda, \varepsilon)=\left\{\sigma \in \Sigma_{n}:\|\sigma\|=\sup _{\varphi \in \Lambda}|\sigma(\varphi)|_{(\varphi)}<\varepsilon<1\right\},
$$

аналогично определяется $\Sigma_{n}^{\prime}(\Lambda, \varepsilon)$. Обозначим через $\mathscr{L}_{0}^{n}(\varepsilon)$ пространство отображений $\mathscr{H}: \Sigma_{n}^{\prime}(\Lambda, \varepsilon) \rightarrow \Sigma_{n}(\Lambda, \varepsilon)$, имеющих константу Липшица $\operatorname{Lip}(\mathscr{H}) \leqslant 1$ и удовлетворяющих условию $\mathscr{H}(0)=0$. Для фиксированного $\mathscr{H} \in \mathscr{L}_{0}^{n}(\varepsilon)$ множество точек вида $(\mathscr{H} \sigma+\sigma+i)(\varphi), \varphi \in \Lambda$, где $\sigma \in \Sigma_{n}^{\prime}(\Lambda, \varepsilon), i$ - вложение $\Lambda \rightarrow W^{1}$, под действием $F_{t}$ переходит в множество $F_{t}((\mathscr{H} \sigma+\sigma+i)(\varphi)), \varphi \in \Lambda$. Условие инвариантности этого множества можно записать в виде

$\pi_{n}(\varphi)\left[F_{t}(\mathscr{H} \sigma+\sigma+i) F_{t}^{-1}-i\right](\varphi)=\mathscr{H}\left\{\pi_{n}^{\prime}(\varphi)\left[F_{t}(\mathscr{H} \sigma+\sigma+i) F_{t}^{-1}-i\right](\varphi)\right\}$, $\varphi \in \Lambda$. Преобразуем это соотношение при помощи формулы конечных приращений:

$$
\begin{aligned}
& \pi_{n}(\varphi)\left[\left(\mathscr{D}_{\varphi_{t}} F_{t}\right)(\mathscr{H} \sigma+\sigma)+\alpha_{t}(\mathscr{H}, \sigma)(\mathscr{H} \sigma+\sigma)\right]\left(\varphi_{t}\right) \\
& \quad=\mathscr{H}\left\{\pi_{n}^{\prime}(\varphi)\left[\left(\mathscr{D}_{\varphi_{t}} F_{t}\right)(\mathscr{H} \sigma+\sigma)+\alpha_{t}(\mathscr{H}, \sigma)(\mathscr{H} \sigma+\sigma)\right]\left(\varphi_{t}\right)\right\},
\end{aligned}
$$

где $\varphi_{t}=F_{t}^{-1}(\varphi), \alpha_{t}(\mathscr{H}, \sigma) \in \operatorname{End}\left(\Sigma_{c}(\Lambda)\right)$ и определяется формулой

$$
\begin{aligned}
& \alpha_{t}(\mathscr{H}, \sigma) \sigma_{1}\left(\varphi_{t}\right) \\
&=\int_{0}^{t}\left(\mathscr{D}_{\xi}(\mathscr{H} \sigma+\sigma)\left(\varphi_{t}\right)+\varphi_{t} F_{t}\right) \sigma_{1}\left(\varphi_{t}\right) d \xi-\left(\mathscr{D}_{\varphi_{t}} F_{t}\right) \sigma_{1}\left(\varphi_{t}\right) .
\end{aligned}
$$


В силу теоремы 1 и формулы (18) существуют константы $A_{i}>0, i=1,2,3$, такие, что при $t \in[0,1], \sigma, \sigma_{1}, \sigma_{2} \in \Sigma_{n}^{\prime}(\Lambda, \varepsilon)$

$$
\begin{aligned}
\left\|\alpha_{t}(\mathscr{H}, \sigma)\right\| & \leqslant A_{1}\|\mathscr{H} \sigma+\sigma\|, & & \mathscr{H} \in \mathscr{L}_{0}^{n}(\varepsilon) \\
\left\|\alpha_{t}\left(\mathscr{H}_{1}, \sigma\right)-\alpha_{t}\left(\mathscr{H}_{2}, \sigma\right)\right\| & \leqslant A_{2}\left\|\left(\mathscr{H}_{1}-\mathscr{H}_{2}\right) \sigma\right\|, & & \mathscr{H}_{1}, \mathscr{H}_{2} \in \mathscr{L}_{0}^{n}(\varepsilon) ; \\
\left\|\alpha_{t}\left(\mathscr{H}, \sigma_{1}\right)-\alpha_{t}\left(\mathscr{H}, \sigma_{2}\right)\right\| & \leqslant A_{3}\left\|\sigma_{1}-\sigma_{2}\right\|, & & \mathscr{H} \in \mathscr{L}_{0}^{n}(\varepsilon) .
\end{aligned}
$$

В силу первого неравенства леммы 3 соотношение (17) можно при достаточно малых $t>0$ преобразовать к виду

$$
\begin{aligned}
& \mathscr{H} \sigma(\varphi)=\left[\pi_{n}(\varphi)\left(\mathscr{D}_{\varphi} F_{t}\right)\right]^{-1} \\
& \quad \times\left\{\mathscr{H}\left[\pi_{n}^{\prime}(\varphi)\left(\mathscr{D}_{\varphi} F_{t}\right)(\mathscr{H} \sigma+\sigma)(\varphi)+\pi_{n}^{\prime}(\varphi) \alpha_{t}(\mathscr{H}, \sigma)(\mathscr{H} \sigma+\sigma)(\varphi)\right]\right. \\
& \left.-\pi_{n}(\varphi) \alpha_{t}(\mathscr{H}, \sigma)(\mathscr{H} \sigma+\sigma)(\varphi)-\pi_{n}(\varphi)\left(\mathscr{D}_{\varphi} F_{t}\right) \sigma(\varphi)\right\} \equiv \mathscr{F}_{t}^{n}(\mathscr{H}) \sigma(\varphi) .
\end{aligned}
$$

Очевидно, $\mathscr{F}_{t}^{n}(\mathscr{H})(0)=0$. Оценим константу Липшица отображения $\mathscr{F}_{t}^{n}(\mathscr{H})$ при $\mathscr{H} \in \mathscr{L}_{0}^{n}(\varepsilon)$. Для этого воспользуемся леммой 3 , неравенствами $(19)$ и определением пространства $\mathscr{L}_{0}^{n}(\varepsilon)$. В результате получим $\operatorname{Lip}\left(\mathscr{F}_{t}^{n}(\mathscr{H})\right) \leqslant R_{0}(n, \varepsilon, t), R_{0}(n, \varepsilon, t)=\left[e^{\varkappa_{n}^{+} t}+\mu_{1} t+\nu_{1} \varepsilon\right]\left[e^{\varkappa_{n}^{-} t}-h_{1} t\right]^{-1}$, где $\varkappa_{n}^{+}=\sup _{\varphi \in \Lambda}\left\{\lambda_{n+1}(\varphi)-\gamma \lambda_{0}^{-1}\right\}, \varkappa_{n}^{-}=\inf _{\varphi \in \Lambda}\left\{\lambda_{n}(\varphi)-\gamma \lambda_{0}^{-1}\right\}$, a константы $\mu_{1}>0, h_{1}>0, \nu_{1}>0$ выражаются через $A_{k}, c_{i}, k=1,2,3$, $i=1,2,3,4$. Введем на множестве $\mathscr{L}_{0}^{n}(\varepsilon)$ метрику:

$$
\left\|\mathscr{H}_{1}-\mathscr{H}_{2}\right\|=\sup _{\sigma \in \Sigma_{n}^{\prime}(\Lambda, \varepsilon)}\left\|\mathscr{H}_{1}(\sigma)-\mathscr{H}_{2}(\sigma)\right\| .
$$

По аналогии с вьводом оценки константы Липшица $\operatorname{Lip}\left(\mathscr{F}_{t}^{n}(\mathscr{H})\right)$ можно получить неравенство $\left\|\mathscr{F}_{t}^{n}\left(\mathscr{H}_{1}\right)-\mathscr{F}_{t}^{n}\left(\mathscr{H}_{2}\right)\right\| \leqslant R_{1}(n, \varepsilon, t)\left\|\mathscr{H}_{1}-\mathscr{H}_{2}\right\|$, где $R_{1}(n, \varepsilon, t)=\left[e^{\varkappa_{n}^{+} t}+\mu_{2} t+\nu_{2} \varepsilon\right]\left[e^{\varkappa_{n}^{-} t}-h_{2} t\right]^{-1}$, константы $\mu_{2}>0, \nu_{2}>0$, $h_{2}>0$ выражаются через $A_{k}, c_{i}, k=1,2,3, i=1,2,3,4$.

Выберем $\delta>0$ столь малым, что $1-s \leqslant e^{-s} \leqslant 1-s+2^{-1} s^{2}$ при $0 \leqslant s \leqslant \delta$. Пусть для $n \in \mathbb{N} \varepsilon_{n}=t_{n}=\delta\left|\varkappa_{1}^{+}\right| \cdot\left|\varkappa_{n}^{+}\right|^{-2}$, тогда $R_{i}\left(n, \varepsilon_{n}, t_{n}\right)<1$, $i=0,1$, если $M \in \mathbb{N}$ столь велико, что при $n \geqslant M$

$$
\varkappa_{n}^{+}-\varkappa_{n}^{-}+\max _{i}\left\{\mu_{i}+\nu_{i}+h_{i}\right\}+2^{-1} \delta\left|\varkappa_{1}^{+}\right|<0 .
$$

Следовательно, при $n \geqslant M$ отображение $\mathscr{F}_{t_{n}}^{n}: \mathscr{H} \mapsto \mathscr{F}_{t_{n}}^{n}(\mathscr{H})$ переводит пространство $\mathscr{L}_{0}^{n}(\varepsilon)$ в себя и является сжимающим. Поэтому у него существует единственная неподвижная притягивающая точка $\mathscr{H}_{n} \in \mathscr{L}_{0}^{n}(\varepsilon)$.

Выберем $n \geqslant M$ и рассмотрим $\operatorname{graph}\left(\mathscr{H}_{n}\right)-$ график отображения $\mathscr{H}_{n}$. Определим семейство подмножеств $W_{\varepsilon_{n}}^{s}(\varphi), \varphi \in \Lambda$, следуюшим образом:

$$
W_{\varepsilon_{n}}^{s}(\varphi)=\left\{\varphi+\psi: \psi \in \operatorname{graph}\left(\mathscr{H}_{n}\right) \cap T_{\varphi} W^{1}\right\} .
$$


Семейство (21) есть $C^{0}$-непрерывное семейство $C^{0}$-подмногообразий, для которого вьполняется утверждение пункта 2 теоремы 2 . Инвариантность семейства подмногообразий $(21)$ вытекает из соотношения $\mathscr{F}_{t_{n}}^{n}\left(\mathscr{H}_{n}\right)=\mathscr{H}_{n}$, полугруппового свойства семейства отображений $F_{t}, t \geqslant 0$, и непрерывности этого семейства по $t$ (теорема 1$)$. При больших $n$ натуральный логарифм константы Липшица отображения $\left[\mathscr{F}_{t_{n}}^{n}\right]^{m(n)}$, где $m(n)$ есть целая часть числа $t_{n}^{-1}$, оценивается сверху величиной

$$
\begin{aligned}
-\left(1+O\left(\left|\varkappa_{n}^{+}\right|^{-1}\right)\right) \mid \varkappa_{n}^{+}-\varkappa_{n}^{-} & \\
& +\max _{i}\left\{\mu_{i}+\nu_{i}+h_{i}\right\}+2^{-1} \delta\left|\varkappa_{1}^{+}\right| \mid \ln \left(e+O\left(\left|\varkappa_{n}^{+}\right|^{-1}\right)\right) .
\end{aligned}
$$

Следовательно, выполняется утверждение пункта 4 теоремы 2 .

Покажем, что семейство (21) есть $C^{1}$-непрерывное семейство $C^{1}$-подмногообразий. Рассмотрим $\Omega\left(\varepsilon_{n}\right)$ - множество непрерьвных отображений $\mathscr{T}: \Sigma_{n}^{\prime}\left(\Lambda, \varepsilon_{n}\right) \rightarrow \operatorname{Hom}\left(\Sigma_{n}^{\prime}, \Sigma_{n}\right)$ таких, что $\|\mathscr{T}(\sigma)\| \leqslant 1$, здесь $\sigma \in \Sigma_{n}^{\prime}\left(\Lambda, \varepsilon_{n}\right), \operatorname{Hom}\left(\Sigma_{n}^{\prime}, \Sigma_{n}\right)$ - банахово пространство непрерьвных линейных отображений $\Sigma_{n}^{\prime}$ в $\Sigma_{n}$. Определим отображение $\mathscr{K}_{t_{n}}^{n}: \mathscr{L}_{0}^{n}\left(\varepsilon_{n}\right) \times$ $\Omega\left(\varepsilon_{n}\right) \rightarrow \mathscr{L}_{0}^{n}\left(\varepsilon_{n}\right) \times \Omega\left(\varepsilon_{n}\right)$ посредством формул

$$
\begin{aligned}
& \left\{p_{1}\left[\mathscr{K}_{t_{n}}^{n}(\mathscr{H}, \mathscr{T})\right]\right\} \sigma(\varphi)=\left[\mathscr{F}_{t_{n}}^{n}(\mathscr{H}) \sigma\right](\varphi), \\
& \left\{p_{2}\left[\mathscr{K}_{t_{n}}^{n}(\mathscr{H}, \mathscr{T})\right] \sigma\right\} \theta(\varphi)=\left[\pi_{n}(\varphi)\left(\mathscr{D}_{\varphi} F_{t_{n}}\right)\right]^{-1} \\
& \quad \times\left\{\mathscr{T}\left(\sigma_{1}\right)\left[\pi_{n}^{\prime}(\varphi)\left(\mathscr{D}_{\varphi} F_{t_{n}}\right)(\mathscr{T}(\sigma)+\mathrm{id})\right] \theta(\varphi)-\pi_{n}(\varphi)\left(\mathscr{D}_{\varphi} F_{t_{n}}\right) \theta(\varphi)\right\}
\end{aligned}
$$

где $\sigma \in \Sigma_{n}^{\prime}\left(\Lambda, \varepsilon_{n}\right), \sigma_{1}(\varphi)=\pi_{n}^{\prime}(\varphi)\left(\mathscr{D}_{\varphi} F_{t_{n}}\right)(\mathscr{H} \sigma+\sigma)(\varphi), \varphi \in \Lambda, p_{i}\left(x_{1}, x_{2}\right)$ $=x_{i}, i=1,2$ - канонические проекции. Отображение $\mathscr{K}_{t_{n}}^{n}$ построено так, что $\left(\mathscr{K}_{t_{n}}^{n}\right)^{m}(\mathscr{H}, \mathscr{D} \mathscr{H}) \sigma=\left(\left(\mathscr{F}_{t_{n}}^{n}\right)^{m}(\mathscr{H}) \sigma, \mathscr{D}_{\sigma}\left(\mathscr{F}_{t_{n}}^{n}\right)^{m}(\mathscr{H})\right)$, здесь $\mathscr{D} \mathscr{H}$ - касательное отображение $\mathscr{D} \mathscr{H}: \sigma \mapsto \mathscr{D}_{\sigma} \mathscr{H}$.

По аналогии с вьводом оценок для отображения $\mathscr{F}_{t_{n}}^{n}$ можно показать, что существует константа $\mu_{3}>0$, для которой

$$
\left\|\left\{p_{2}\left[\mathscr{K}_{t_{n}}^{n}(\mathscr{H}, \mathscr{T})\right] \sigma\right\}\right\| \leqslant\left[e^{\varkappa_{n}^{+} t_{n}}+\mu_{3} t_{n}\right]\left[e^{\varkappa_{n}^{-} t_{n}}-\mu_{3} t_{n}\right]^{-1} .
$$

В силу этого неравенства отображение $\mathscr{K}_{t_{n}}^{n}$ определено корректно при достаточно больших $n$.

В соответствии со стандартной схемой техники преобразования графиков для доказательства дифференцируемости и $C^{1}$-непрерывности семейства (21) достаточно показать, что отображение $\mathscr{K}_{t_{n}}^{n}$ имеет единственную притягивающую неподвижную точку. Воспользуемся принципом сжимающих отображений для отображения расслоения (см. [8, $\S 5.2]$ ). Согласно этому принципу в данном случае достаточно показать, что при достаточно больших $n$ константа Липшица отображения $p_{2} \mathscr{K}_{t_{n}}^{n}: \Omega\left(\varepsilon_{n}\right) \rightarrow \Omega\left(\varepsilon_{n}\right)$ меньше единицы при любом $\mathscr{H} \in \mathscr{L}_{0}^{n}\left(\varepsilon_{n}\right)$. Но это нетрудно сделать по аналогии с выводом оценок для отображения $\mathscr{F}_{t_{n}}^{n}[3]$. Таким образом, пункт 1 теоремы 2 доказан. 
Убедимся в справедливости пункта 5 утверждения теоремы 2 . Пусть подпространства $H_{n}$ и $H_{n}^{\prime}$ натянуты, соответственно, на семейства собственных функций $\left\{v_{1}, \ldots, v_{n}\right\}$ и $\left\{v_{k}, k>n\right\}$ оператора $L$. Операторы проектирования $\pi_{n}$ и $\pi_{n}^{\prime}$ определяются прямой суммой $W^{1}=H_{n} \oplus H_{n}^{\prime}$. Из формул (3)-(6) вытекает следуюшее соотношение между собственными функциями операторов $L$ и $L_{\varphi}$ :

$$
v_{n}(\varphi, x)=\Phi(\varphi, x)\left[v_{n}(x)+O\left(n^{-1}\right)\right], \quad n \in \mathbb{N},
$$

где

$$
\Phi(\varphi, x)=\exp \left\{-2^{-1} \int_{0}^{x}\left[f_{u_{x}}(\varphi, s)(d(s))^{-1}\right] d s\right\} .
$$

Рассмотрим ограничение $\pi_{n}^{\prime}$ на подпространство $H_{n}^{\prime}(\varphi)$ при больших $n$. Предположим, что существует последовательность $u_{m}=\sum_{k>n_{m}} c_{k}^{(m)} \times$ $v_{k}\left(\varphi_{m}, x\right), n_{m} \rightarrow \infty, \varphi_{m} \in \Lambda$, такая, что $\left|u_{m}\right|=1$, а $\delta_{m}=\left|\pi_{m}^{\prime} u_{m}\right| \rightarrow 0$ при $m \rightarrow \infty$. В таком случае

$$
u_{m}=\sum_{k=1}^{n_{m}} \alpha_{k}^{(m)} v_{k}(x)+\Delta_{m}(x)
$$

где $\Delta_{m}(x) \in H_{n_{m}}^{\prime}$. В силу (22) справедливо соотношение

$$
\sum_{k=1}^{n_{m}} \alpha_{k}^{(m)} v_{k}(x)=\Phi\left(\varphi_{m}, x\right) \sum_{k>n_{m}} c_{k}^{(m)}\left(v_{k}(x)+O\left(k^{-1}\right)\right)-\Phi\left(\varphi_{m}, x\right) \Delta_{m}(x),
$$

из которого, считая неизвестным $\alpha^{(m)}=\left(\alpha_{1}^{(m)}, \ldots, \alpha_{n_{m}}^{(m)}\right)$, получаем систему линейных уравнений $A_{m} \alpha^{(m)}=\beta^{(m)}$, где

$$
\begin{aligned}
\beta^{(m)} & =\left(\beta_{1}^{(m)}, \ldots, \beta_{n_{m}}^{(m)}\right), \\
\beta_{s}^{(m)} & =\sum_{k>n_{m}} c_{k}^{(m)}\left(O\left(k^{-1}\right), v_{s}(x)\right), \\
\left(A_{m} \alpha^{(m)}\right)_{s} & =\sum_{k=1}^{n_{m}} \alpha_{k}^{(m)}\left(\Phi^{-1}\left(\varphi_{m}, x\right) v_{k}(x), v_{s}(x)\right),
\end{aligned}
$$

$s=1, \ldots, n_{m}$. Евклидова норма $\left\|\beta^{(m)}\right\| \rightarrow 0$ при $m \rightarrow \infty$. Для любых $\alpha_{i}$, $i=1, \ldots, n_{m}, \varphi_{m} \in \Lambda$ вьполняется оценка

$$
\begin{aligned}
\sum_{i, j=1}^{n_{m}} \alpha_{i} \alpha_{j}\left(\Phi^{-1}(\varphi, x)\right. & \left.v_{i}(x), v_{j}(x)\right) \\
= & \left|\Phi^{-1 / 2}(\varphi, x) \sum_{i=1}^{n_{m}} \alpha_{i} v_{i}(x)\right|^{2} \geqslant \gamma_{1}^{2} \sum_{i=1}^{n_{m}} \alpha_{i}^{2},
\end{aligned}
$$


где константа $\gamma_{1}>0$ не зависит от $m$ и $\varphi$. Следовательно, $\left\|\alpha^{(m)}\right\| \rightarrow 0$, что противоречит соотношению $1=\left\|\alpha^{(m)}\right\|^{2}+\delta_{m}^{2}$. Таким образом, для некоторой не зависящей от $\varphi \in \Lambda, n \in \mathbb{N}$ константы $\Delta>0$ при достаточно больших $n$ имеет место неравенство $\left|\pi_{n}^{\prime} u\right| \geqslant \Delta|u|, u \in H_{n}^{\prime}(\varphi)$. Аналогично доказьвается неравенство $\left|\pi_{n}^{\prime} u\right|_{1} \geqslant \omega|u|_{1}, u \in H_{n}^{\prime}(\varphi)$, где $\omega>0$ не зависит от $\varphi$ и $n$. Следовательно, при достаточно больших $n$ ограничение $\pi_{n}^{\prime}$ на $H_{n}^{\prime}(\varphi)$ есть изоморфизм, а подпространство $H_{n}^{\prime}(\varphi)$ есть график непрерывного линейного отображения $G_{\varphi, n}: H_{n}^{\prime} \rightarrow H_{n}$, причем $\left|G_{\varphi, n}\right|_{1}<\omega^{-1}$. Но подпространство $H_{n}^{\prime}(\varphi)$ является касательньп подпространством к подмногообразию $W_{\varepsilon_{n}}^{s}(\varphi)$ в точке $\varphi \in \Lambda$. Следовательно, из-за компактности $\Lambda$, непрерьвной дифференцируемости $W_{\varepsilon_{n}}^{s}(\varphi)$ и формулы (21) для достаточно больших $n$ найдутся не зависяшие от $\varphi \in \Lambda$ числа $0<\delta_{n} \leqslant \varepsilon_{n}$ такие, что

$$
W_{\delta_{n}}^{s}(\varphi)=\left\{\varphi+\psi: \psi \in \operatorname{graph}\left(\bar{G}_{\varphi, n}\right)\right\}
$$

Отображения $\bar{G}_{\varphi, n}: H_{n}^{\prime} \rightarrow H_{n}$ определены в окрестности нуля и для любого $\theta$ из области определения

$$
\left|\mathscr{D}_{\theta} \bar{G}_{\varphi, n}\right|_{1} \leqslant \omega_{1}
$$

Константа $\omega_{1}>0$ не зависит от $\varphi \in \Lambda$ и определяется величинами $\omega, \beta_{1}$, $\beta_{2}$ (величины $\beta_{1}, \beta_{2}$ определены в лемме 2 ).

Пусть $\varphi_{1}, \varphi_{2} \in W_{\varepsilon_{n}}^{s}(\varphi) \cap \Lambda$. Докажем, что $\varphi_{1}=\varphi_{2}$. Благодаря пункту 4 теоремы 2 без ограничения общности можно считать, что $\varphi_{1}$, $\varphi_{2} \in W_{\delta_{n}}^{s}(\varphi) \cap \Lambda$. Следовательно, в силу (23) достаточно доказать соотношение $\pi_{n}^{\prime}\left(\varphi_{1}-\varphi_{2}\right)=0$ для некоторого $n \in \mathbb{N}$. Рассмотрим $u=u_{1}-u_{2}$, где $u_{i}(t)=F_{t}\left(\varphi_{i}\right), i=1,2$. Очевидно, $\pi_{n}^{\prime} u=v$ есть решение задачи Коши с однородными краевыми условиями I рода:

$$
v_{t}=-L v+\pi_{n}^{\prime}\left(B\left(u_{1}\right)-B\left(u_{2}\right)\right)
$$

Перенесем первое слагаемое из правой части (25) в левую и умножим полученное соотношение на себя в $W$. В результате получим

$$
\left|v_{t}\right|^{2}+\frac{d}{d t}|v|_{1}^{2}+|v|_{2}^{2}=\left|\pi_{n}^{\prime}\left(B\left(u_{1}\right)-B\left(u_{2}\right)\right)\right|^{2}
$$

Так как $v \in H_{n}^{\prime}$, то $|v|_{2}^{2} \geqslant \lambda_{n+1}^{2}|v|_{1}^{2}$. Для правой части (26) воспользуемся инвариантностью множества $\Lambda$, его ограниченностью в $W^{k}, k \in \mathbb{N}$, формулой конечных приращений и теоремой вложения для элементов $W^{1}$. В итоге, для некоторой константы $\omega_{2}>0$ получаем

$$
\left|\pi_{n}^{\prime}\left(B\left(u_{1}\right)-B\left(u_{2}\right)\right)\right|^{2} \leqslant \omega_{2}^{2}|u|_{1}^{2}=\omega_{2}^{2}\left(|v|_{1}^{2}+\left|\pi_{n} u\right|_{1}^{2}\right)
$$


В силу пункта 4 теоремы 2 существует $T>0$ такое, что $F_{t}\left(W_{\delta_{n}}^{s}(\varphi)\right) \subset$ $W_{\delta_{n}}^{s}\left(F_{t}(\varphi)\right), t>T$. Следовательно, из $(23),(24)$ получаем $\left|\pi_{n} u\right|_{1} \leqslant \omega_{1}|v|_{1}$, $t>T$. Таким образом, из (26) вытекает неравенство для $t>T$

$$
\frac{d}{d t}|v|_{1}^{2}+\lambda_{n+1}^{2}|v|_{1}^{2}-\omega_{2}^{2}\left(1+\omega_{1}^{2}\right)|v|_{1}^{2} \leqslant 0
$$

Если $n$ столь велико, что $\lambda_{n+1}^{2}>\omega_{2}^{2}\left(1+\omega_{1}^{2}\right)$, то из (28) и ограниченности $\Lambda$ в $W^{1}$ следует $v \equiv 0$. Теорема доказана.

ЗАмЕЧАниЕ 1 . Пункт 5 теоремы 2 можно считать нелинейным аналогом утверждений $\S 3$ работы [7].

ЗАмечАние 2. Рассуждения настоящей заметки обобщаются на случай систем уравнений вида

$$
\begin{aligned}
u_{t} & =-L u+f\left(u, u_{x}, x, t, X\right), \\
\frac{d X}{d t} & =g(X, l(u), t) .
\end{aligned}
$$

Уравнение (29) есть квазилинейное параболическое уравнение типа (1), а (30) - система обыкновенных дифференциальных уравнений. Функционал $l(u)$ - гладкий.

Физико-энергетический институт

Поступило

г. Обнинск 04.04 .91

\section{СПИСОК ЦИТИРОВАННОЙ ЛИТЕРАТУРЫ}

[1] Ладыженская О. А. Краевые задачи математической физики. М.: Наука, 1973.

[2] Mane R. Reduction of semilinear parabolic equation to finite dimensional $C^{1}$-flows // Lect. Notes in Math. 1977. V. 597. P. 361-378.

[3] Камаев Д. А. О гипотезе Хопфа для одного класса уравнений химической кинетики // Записки научн. семинаров ЛОМИ. 1981. Т. 110. С. 57-73.

[4] Constantin P., Foias C., Nicolaenco B., Temam R. Integral manifolds and inertial manifolds for dissipative partial differential equations // Appl. Math. Sci. 1989. V. 70.

[5] Л адыженская О. А., Солонников В. А. О принципе линеаризации и инвариантных многообразиях для задач математической гидродинамики // Записки научн. семинаров ЛОМИ. 1973. Т. 38. С. 46-93.

[6] Левитан Б. М., Саргсян И. С. Введение в спектральную теорию. М.: Наука, 1970.

[7] Л адыженская О.А. О динамической системе, порождаемой уравнениями Навье-Стокса // Записки научн. семинаров ЛОМИ. 1972. Т. 27. С. 91-115.

[8] Нитецки З. Введение в дифференциальную динамику. М.: Мир, 1975. 